\title{
A Biomechanical Comparison of Tendon Repair with a Knotless Barbed Suture and a Conventional Monofilament Suture Material: An ex-vivo Animal Experiment
}

\author{
Halil Can Gemalmaz (1) \\ Acıbadem Mehmet Ali Aydınlar University Faculty of Medicine, Department of Orthopedics and Traumatology, İstanbul, Turkey
}

Cite this article as: Gemalmaz HC. A Biomechanical Comparison of Tendon Repair with a Knotless Barbed Suture and a Conventional Monofilament Suture Material: An ex-vivo Animal Experiment. JAREM 2020;10(1): 1-5

\begin{abstract}
Objective: Our aim is to compare the ultimate tensile strength and stiffness of a 2/0 barbed suture and a 3/0 polypropylene monofilament suture in a porcine tendon repair model.

Methods: Sixteen porcine Achilles tendons were transected and separated into two groups. In group I tendons were repaired with a modified knotless four-strand Kessler technique using a 2/0 V-Loc barbed suture. In group II tendons were repaired with a four-strand Kessler technique using a 3/0 monofilament conventional suture and knots were tied. All specimens were biomechanically tested for ultimate tensile strength (UTS) and stiffness. Mode of failure was also noted.

Results: Five specimens in group I failed by stripping of the suture from the tendon tissue, three failed due to suture breakage. In group II two out of eight tendons failed by stripping of the suture and remaining six failed by suture breakage. Median UTS value was found to be $85.96 \mathrm{~N}$ (range: 63.24 ) in group I and $64.29 \mathrm{~N}$ (range: 56.84 ) in group II. Median stiffness value of the samples in group I was found to be $5.67 \mathrm{~N} / \mathrm{mm}$ (range: 4.32 ) and in group II it was found to be $4.53 \mathrm{~N} / \mathrm{mm}$ (range: 6.23). The statistical analysis of UTS and stiffness values revealed no significant difference between the groups ( $p=0.17$ and $p=0.56$ respectively).

Conclusion: A knotless Kessler tendon repair made with a 2/0 barbed suture is biomechanically equivalent to a knotted Kessler tendon repair made with a 3/0 conventional polypropylene suture in ex-vivo conditions.

Keywords: Tendon repair, barbed suture, Kessler
\end{abstract}

\section{INTRODUCTION}

The ideal tendon repair should be strong enough to enable early range of motion exercises, should be easily performed, should minimize injury to tendon vasculature and should not be bulky to facilitate smooth gliding of the tendon (1-3). However, the higher the number of strands that cross the tendon, the more manipulation needed to put them in the tendons and the greater number of knots needed to tie them off which eventually results in a bulky construct.

The recently introduced barbed sutures have properties which render them very different from conventional sutures and yet they have not been extensively tested for tendon repair scenarios. The evenly distributed micro spikes on the barbed suture helps it with unidirectional anchoring all across the suture trajectory, thus they do not require complex trajectories and knots for tissue grasping and friction $(4,5)$. These spikes generate homogenous and steady friction against slippage throughout suture trajectory in the opposite direction of the suture introduction. Increased tissue friction generated by this behavior of the barbed suture represents itself as a theoretical advantage for tendon repair scenarios since it eliminates the necessity for complex suture trajectories, locking loops and tying knots which are all proven to be mechanical and inflammatory stress risers $(4,5)$. Yet, the same spikes also represent

ORCID IDs of the authors: H.C.G. 0000-0002-5741-199X 
a mechanical disadvantage. The spikes in a barbed suture are created by generating cuts on the body of a monofilament suture which decreases the effective diameter of the barbed suture. Therefore, these evenly distributed cuts through the body of the suture create mechanical stress risers which render them weaker than a conventional monofilament suture of the same material and diameter (6-8).

All these differences between the barbed sutures and conventional monofilament suture materials create a basis for extensive investigation of barbed sutures' role and performance in tendon repair scenarios. Currently, the evidence on barbed suture as a tendon repair device is limited and it is still not clear if their disadvantage of being weaker at the location of spikes are compensated by not being dependent on afore mentioned stress risers. Therefore, in this study, we aim to compare the ultimate tensile strength (UTS) and stiffness of a $2 / 0$ barbed suture and a $3 / 0$ conventional polypropylene monofilament suture in an exvivo porcine Achilles tendon repair model.

\section{METHODS}

\section{Experimental Method}

All ex-vivo specimen used in the study were obtained from animals which were euthanized for endoscopic education purposes at our institution, thus no ethics committee approval was obtained. Sixteen porcine Achilles tendons were surgically extracted from freshly euthanized animals and were fresh frozen in an industrial fridge at -21 centigrade for later use. On the experiment day, the tendons were thawed at room temperature and divided into two groups of 8 tendons. All tendons were cut in the middle with a sharp scalpel blade (Figure 1). The tendons were repaired by two separate (four strand) modified Kessler core sutures (Figure 2a and 2b). In group 1, a 2/0 barbed polypropylene (V-Loc PBT Non-Absorbable Wound Closure Device, Covidien Deutschland $\mathrm{GmbH}$, Neustadt, Germany) was used for the repair (Figure 3) and sutures were left untied. In group II, a 3/0 polypropylene monofilament suture (Prolene, Ethicon, Somerville, N.J., USA) was used and two separate knots were tied. A suture purchase length of $1 \mathrm{~cm}$ was devised for the repairs in both groups.

\section{Biomechanical Test}

A 3kN MTS Acumen 3 testing machine (MTS Corp., Shenzhen, China) was used to assess UTS of the constructs. The both ends of the samples were mounted directly to the clamps of the mechanical testing device making sure that the samples were parallel to the pull direction of the device (Figure 4). After mounting, specimens were axially preloaded with $1 \mathrm{~N}$ of force. The specimens were pulled with a speed of $20 \mathrm{~mm} /$ minute until failure was confirmed on the stress strain graph on the screen of the mechanical testing device or visual conformation of suture failure by breakage or pull out. The mode of failure was also noted for samples.

\section{Statistical Analysis}

Inter group differences for UTS and stiffness were analyzed with the Mann-Whitney $U$ test at $95 \%$ confidence interval, using SPSS
21.0 for Windows (Release 21.0, SPSS Inc, Chicago, IL, USA) computer software.

\section{RESULTS}

Five specimens (62.5\%) in group 1 failed by stripping of suture from the tendon tissue, three (37.5\%) failed due to suture breakage. In group 2, two out of eight tendons (25\%) failed by stripping of the suture and remaining six (75\%) failed by the breakage of which 4 (50\%) were adjacent to the knot.

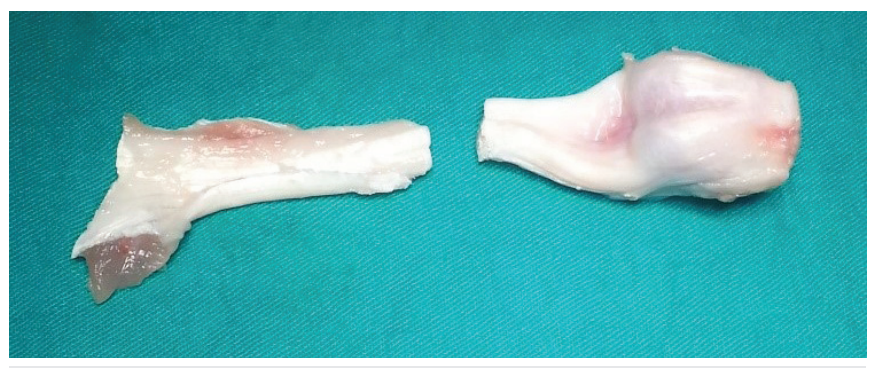

Figure 1. A transected tendon sample
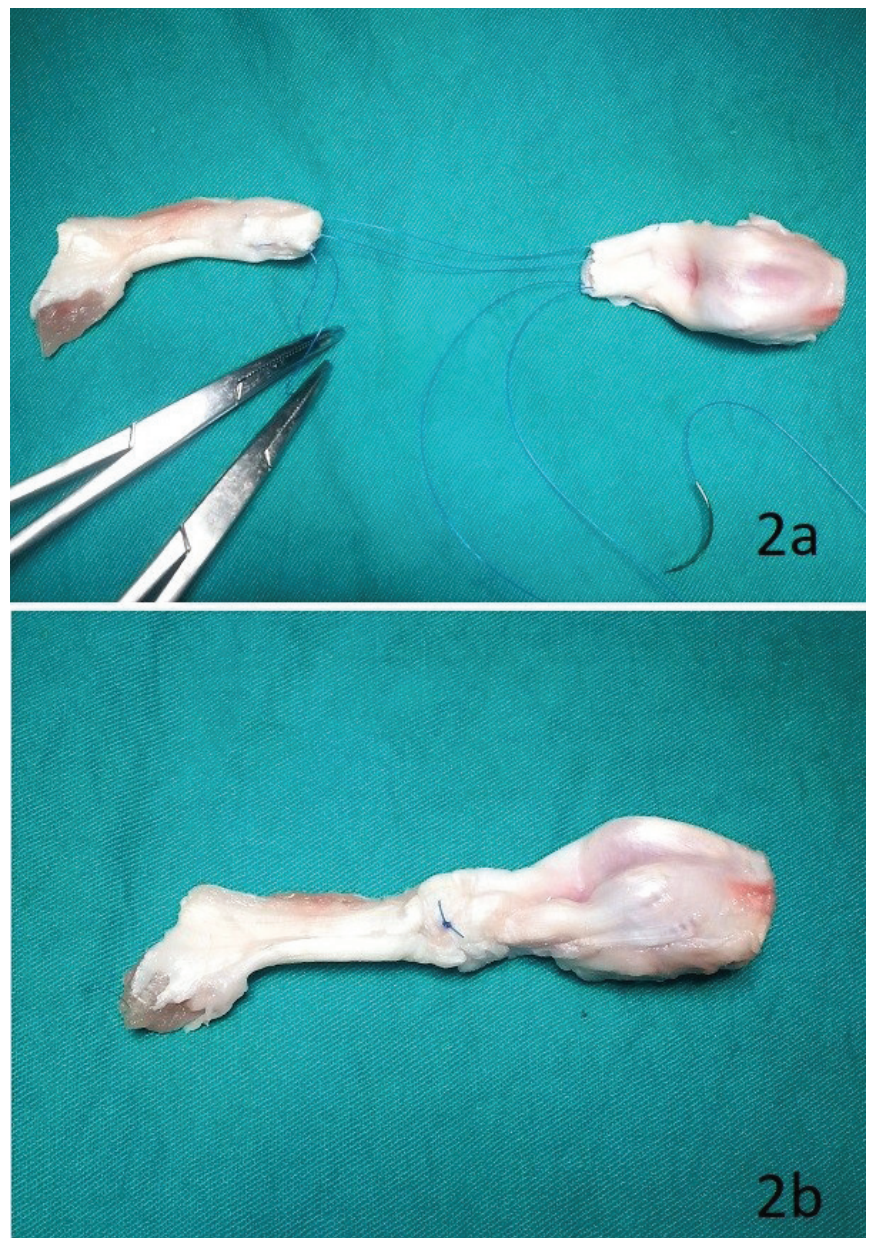

Figure 2 a) Photograph showing application of the dual Kessler sutures to the cut tendon before approximation

Figure 2 b) Photograph showing application of the dual Kessler sutures to the cut tendon after approximation 
between the groups (Mann-Whitney $U$ test: 19, $p=0.17$ two-tailed). The statistical analysis of the stiffness values of the groups with the Mann-Whitney $U$ test also showed that there was no significant difference between these two groups (Mann-Whitney $U$ test: 26.5, $p=0.56$ two-tailed).

\section{DISCUSSION}

The findings of our study provide new data to the literature in the field of research of barbed sutures for tendon repair scenarios. In this biomechanical study, our comparison of a barbed suture and a conventional monofilament suture for UTS and stiffness revealed that there was no statistically significant difference between the groups.

The specification sheets of the barbed sutures and previous studies state that tensile properties of the barbed sutures of a certain diameter are comparable to unbarbed sutures of one size smaller diameter conventional monofilament sutures in a straightpull test as a result of decreased effective diameter caused by the methodology for creating barbs (6-8). Therefore, in this study, we compared a $2 / 0$ barbed suture to a $3 / 0$ monofilament suture. Yet, previous studies prove that repair site thickness of tendons repaired with barbed sutures of one size larger

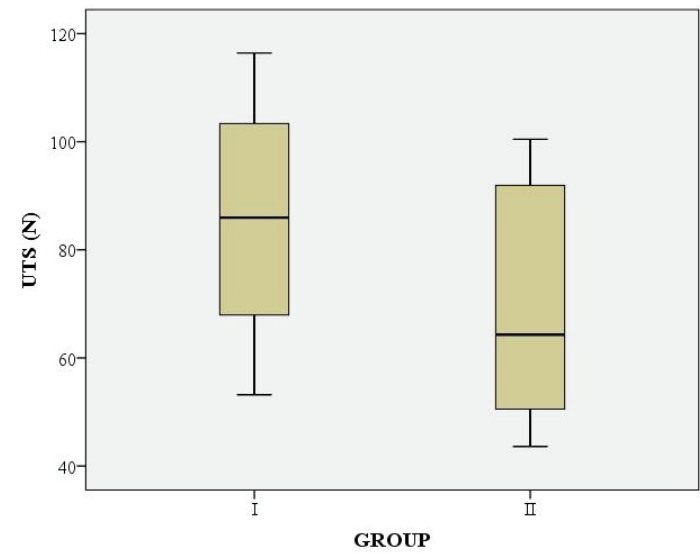

Figure 3. Close up photograph of the V-loc barbed suture depicting micro anchors

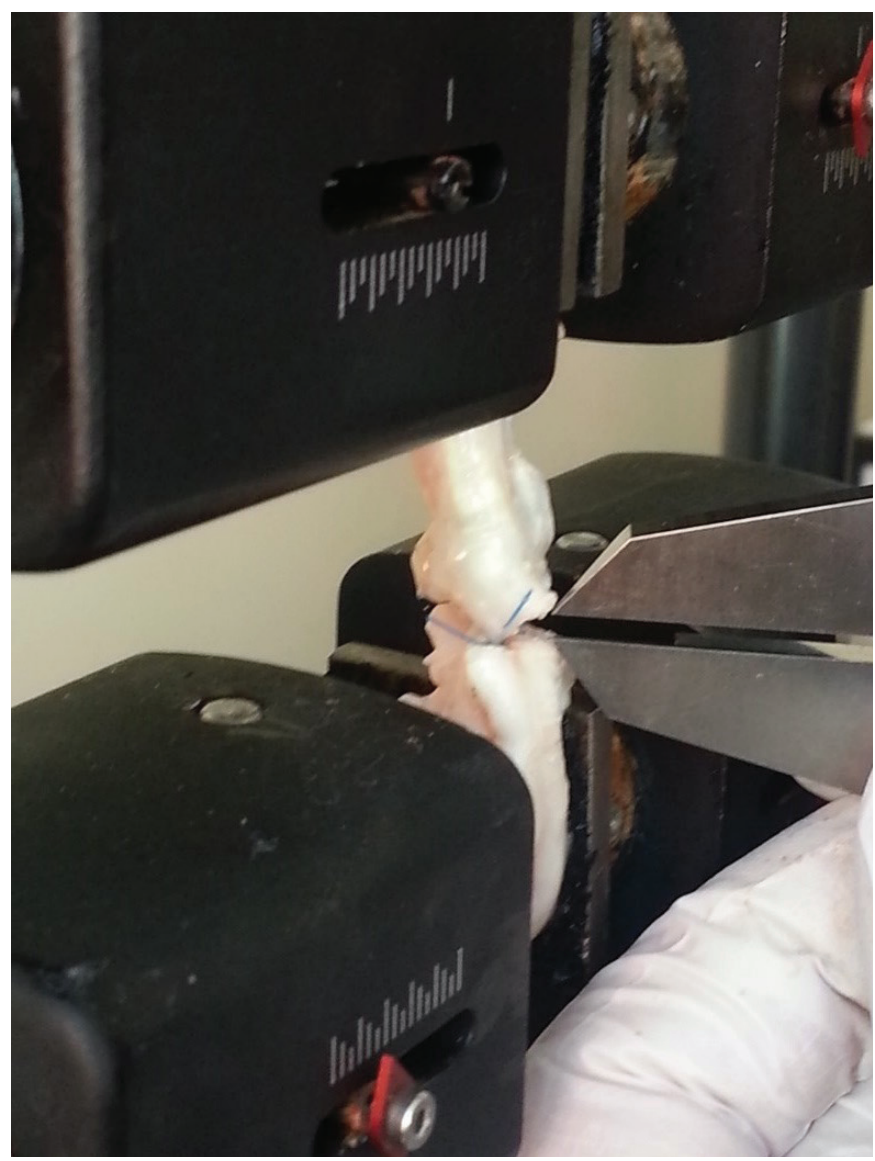

Figure 4. Photograph showing a sample mounted on the mechanical test device for biomechanical testing

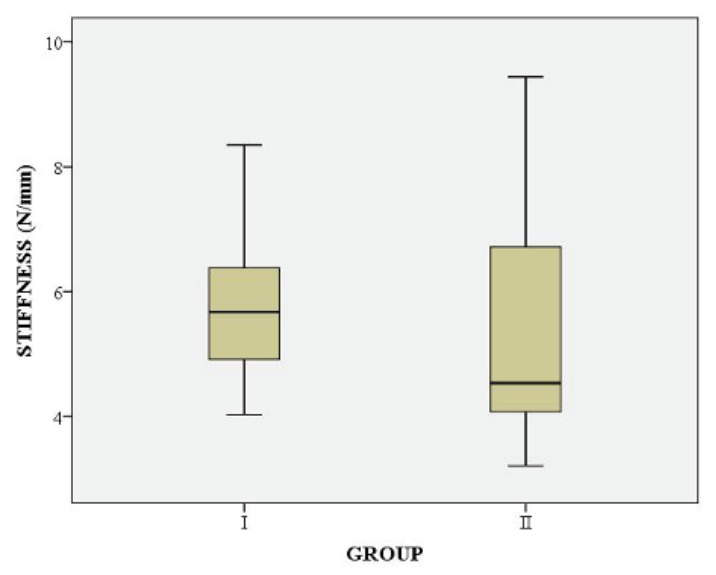

Figure 5, 6: Box-plot charts depicting the minimum-maximum values of UTS and stiffness values for easy visualization of study data 
diameter is comparable or thinner than the tendons repaired with conventional sutures of one size smaller diameter for the fact that they do not require bulky knots to be tied (7-9).

When we compare our findings to the literature, we can see that our findings show parallelism to some similar studies in the literature. The previous studies also do suggest that tendon repair made with the barbed suture which is one size larger by diameter than the compared conventional monofilament suture displays identical UTS and stiffness properties $(6-8,10)$.

Joyce et al. (7) have investigated a novel four strand suture technique with 2/0 barbed suture to a four strand Adelaide technique with $3 / 0$ conventional monofilament suture. Their results showed that the mean ultimate strength of the barbed repairs was $54.51 \mathrm{~N}$ while that of the Adelaide repairs was $53.17 \mathrm{~N}$ and statistical analysis of their data showed no difference for UTS between the groups. On the other hand, their measurements of 2 $\mathrm{mm}$ gap forming force which represents stiffness of the construct favored the barbed group over conventional suture group, which we believe is a result of the new technique they devised for the barbed suture group. In another study by Clemente et al. (8) which investigated a four-strand new technique with $2 / 0$ barbed sutures to two strand modified Kessler technique with $3 / 0$ conventional monofilament sutures, they found identical UTS and $2 \mathrm{~mm}$ gap formation forces when the results were corrected for the number of strands in the repair. Yet the study by Marrero-Amadeo et al. (6) also confirms these findings that $2 / 0$ barbed suture performs biomechanically similar to $3 / 0$ conventional monofilament suture.

Contrary to our findings which showed the barbed suture to be biomechanically equivalent to the conventional monofilament sutures, there are studies which have found them to be inferior and superior to the conventional sutures (9,11-14). Trocchia et al. (11) reported that Kessler tendon repair done with a 2/0 barbed suture was biomechanically inferior to the repair done with a 3/0 non-absorbable braided conventional suture. McClellan et al. (12) investigated the ultimate tensile strengths of three different tendon repairs. In the first group, repairs were made using a 3/0 Ethibond modified Kessler 2-strand repair, in the second group a 3-0 Ethibond modified Savage repair and in the third group a unique knotless 4-0 novel barbed suture. They reported that the Savage and knotless repair groups performed significantly better as compared to the modified Kessler repair group. Peltz et al. (13) compared three groups of different tendon repairs, in which one group consisted of a four-strand Adelaide 3-0 Ticron traditional repair, the other a novel four-strand 3-0 Ticron suture repair and the final group with the same novel four-strand technique as in group 2 but with a 3-0 V-Loc barbed suture instead. Their results indicate significantly better ultimate tensile strengths favoring the barbed V-Loc over the other techniques and sutures.

It is critical that a tendon repair suture should not fail prematurely before reaching strength values close to the suture material's simple straight pull test yield values. Suture failing by pull-out is an example of premature failing since the suture does not break, meaning that the force required to fail is less than the suture material's yield strength. Suture breakage adjacent to the knots is another example of premature failure since knots create a weak point in the filament. As a result, the ideal mode of suture failure is suture breakage in the continuum of the filament where there is no mechanical stress riser.

In our study, five specimens in the barbed suture group failed by stripping of suture from the tendon tissue and the remaining three failed due to suture breakage in the continuum of the filament. In the non-barbed suture group, two out of eight tendons failed by stripping and six by suture breakage of which four were adjacent to the knots. The analysis of the failure modes in our study suggests that Kessler suture technique may not be the most suitable for tendon repair with the barbed suture since most of the specimens $(67.5 \%)$ in this group failed by suture slippage. We believe that modern tenorrhaphy techniques including Kessler have evolved for conventional non-barbed sutures through years of basic and clinical research. Therefore, new research efforts on tendon repair investigating barbed suture's role should concentrate on new techniques which are customized for barbed sutures. Yet, our results confirm that knots do create weak points in the filament forming stress riser areas since four out of eight (50\%) specimens in the non-barbed group failed by breakage adjacent to the knot. Studies with similar methodology to ours confirm similar modes of failure data.

Marrero-Amadeo et al. (6) reported that repairs with conventional monofilament sutures with knots failed $65 \%$ by suture rupture and the remaining repairs failed by the suture knot unraveling, whereas repairs made with the barbed sutures failed $67 \%$ by suture pull-out, and the remaining repairs failed by suture rupture. Joyce et al. (7) also found comparable results about the mode of suture failure. They have reported that the cause of suture failure for the barbed group was breakage in $60 \%$ of the cases and pullout in the remainder. For the non-barbed group, they have reported a suture failure mode of pull out by $60 \%$ and suture breakage by $40 \%$ noting that seven out of eight suture breakage cases occurred adjacent to the knot.

Although we believe that our study fulfills a role in providing additional data on barbed suture's role in the tendon repair scenarios, our study has its own limitations as well. Firstly, the sample size for the groups of our study is the minimum limit for such a study to be relevant. Another limitation to our study is that no measurements of tendon repair site diameter was made, which is often useful to speculate on tendon gliding performance. Next, our study like many other similar ones in the literature compares the biomechanical performance of a barbed suture to a nonbarbed suture with a suture technique which has evolved for non-barbed sutures, thus theoretical advantages of the barbed suture are dwindled by this move. Finally, as being an ex-vivo biomechanical study, our findings lack the strong evidence which can only be provided by randomized controlled in-vivo studies.

\section{CONCLUSION}

Our study confirms some of the previous findings of the literature indicating that the knotless tendon repair made with a one size 
larger diameter barbed suture is biomechanically equivalent to the knotted tendon repair made with a one size smaller diameter conventional monofilament suture in-vitro conditions. With the theoretical advantages of the barbed suture such as easy application, no dependency on complex trajectories, locking loops and knots, these sutures may prove to be beneficial for tendon repair scenarios. Yet, further research with potentially advantageous suture techniques and in-vivo studies are needed to assess barbed suture's role in tendon repair.

Ethics Committee Approval: All ex-vivo specimen used in the study were obtained from animals which were euthanized for endoscopic education purposes at our institution, thus no ethics committee approval was obtained.

Informed Consent: Patient approval has not been obtained as it is performed on animals.

Peer-review: Externally peer-reviewed.

Financial Disclosure: The authors declared that this study has received no financial support.

\section{REFERENCES}

1. Momose T, Amadio PC, Zhao C, Zobitz ME, An KN. The effect of knot location, suture material, and suture size on the gliding resistance of flexor tendons. J Biomed Mater Res 2000; 53: 806-11.

2. Wu YF, Tang JB. Recent developments in flexor tendon repair techniques and factors influencing strength of the tendon repair. J Hand Surg Eur Vol 2014; 39: 6-19.

3. Müller SA, Todorov A, Heisterbach PE, Martin I, Majewski M. Tendon healing: An overview of physiology, biology, and pathology of tendon healing and systematic review of state of the art in tendon bioengineering. Knee Surg Sports Traumatol Arthrosc 2015; 23: 2097-105.
4. Shah A, Rowlands M, Au A. Barbed Sutures and Tendon Repair-a Review. Hand (NY) 2015; 10: 6-15.

5. Shin JY, Kim JS, Roh SG, Lee NH, Yang KM. Biomechanical analysis of barbed suture in flexor tendon repair versus conventional method: Systematic review and meta-analysis. Plast Reconstr Surg 2016; 138 : 666e-74e.

6. Marrero-Amadeo IC, Chauhan A, Warden SJ, Merrell GA. Flexor tendon repair with a knotless barbed suture: a comparative biomechanical study. J Hand Surg Am 2011; 36: 1204-8.

7. Joyce CW, Sugrue C, Chan JC, Delgado L, Zeugolis D, Carroll SM, et al. A barbed suture repair for flexor tendons: a novel technique with no exposed barbs. Plast Reconstr Surg Glob Open 2014; 2: e237.

8. Clemente A, Bergamin F, Surace C, Lepore E, Pugno N. Barbed suture vs conventional tenorrhaphy: biomechanical analysis in an animal model. J Orthop Traumatol 2015; 16: 251-7.

9. Parikh PM, Davison SP, Higgins JP. Barbed suture tenorrhaphy: an ex vivo biomechanical analysis. Plast Reconstr Surg 2009; 124: 1551-8.

10. Zeplin PH, Zahn RK, Meffert RH, Schmidt K. Biomechanical evaluation of flexor tendon repair using barbed suture material: a comparative ex vivo study. J Hand Surg Am 2011; 36: 446-9.

11. Trocchia AM, Aho HN, Sobol G. A re-exploration of the use of barbed sutures in flexor tendon repairs. Orthopedics 2009.

12. McClellan WT, Schessler MJ, Ruch DS, Levin LS, Goldner RD. A knotless flexor tendon repair technique using a bidirectional barbed suture: an ex vivo comparison of three methods. Plast Reconstr Surg 2011; 128: 322e7 e.

13. Peltz TS, Haddad R, Scougall PJ, Gianoutsos MP, Bertollo N, Walsh WR. Performance of a knotless four-strand flexor tendon repair with a unidirectional barbed suture device: a dynamic ex vivo comparison. J Hand Surg Eur Vol 2014; 39: 30-9.

14. Lin TE, Lakhiani C, Lee MR, Saint-Cyr M, Sammer DM. Biomechanical analysis of knotless flexor tendon repair using large-diameter unidirection barbed suture. Hand (NY) 2013; 8: 315-9. 